Reprod. Nutr. Dévelop., 1982, 22 (2), 371-378.

\title{
Recherche de l'origine des fibres nerveuses extra- orthosympathiques innervant l'épiphyse du rat létude du transport rétrograde de la peroxydase de raifort)
}

\author{
Chantal GUÉRILLOT, A. PFISTER, Jacqueline MÜLLER, C. DA LAGE \\ Laboratoire d'Histologie et Embryologie, \\ Faculté de Médecine Necker/Enfants Malades, \\ 156, rue de Vaugirard, 75015 Paris, France.
}

Summary. Determination by feedback transport of horseradish peroxidase of the origin of extra-orthosympathetic nerve fibers innervating the rat pineal gland.

After horseradish peroxidase was injected into the rat pineal gland, isolated and rare labelled perikarya appeared in the central nervous system, particularly in the habenula, colliculi, amygdala, paraventricular and suprachiasmatic nuclei, preoptic area and olfactive. centers. This indicates that the innervation of the pineal gland is not only orthosympathetic but that some stimuli can be directly transmitted from the brain to the pineal stalk.

\section{Introduction.}

Le rôle de l'éclairement sur la rythmicité de la synthèse et de l'excrétion des indolamines par l'épiphyse est connu depuis longtemps. Cette particularité a été expliquée par les relations nerveuses de la glande avec les voies optiques par l'intermédiaire de circuits passant par les bandelettes optiques, le faisceau médian du télencéphale, la moelle, les ganglions cervicaux supérieurs et les nerfs conaires (Kappers, 1960).

D'autres observations montrent que l'activité de la pinéale est également modulée par des influences olfactives (Miline, Devečerski et Krstić, 1963 ; Reiter et Ellison, 1970 ; Dafny, 1977) ou auditives (Dafny, 1977 ; Burks et Dafny, 1977). Des modifications du comportement chez les animaux épiphysectomisés ou recevant des extraits pinéaux (McKinney, Vaughan et Reiter, 1975) ont été mises en évidence. Enfin, l'épiphyse possède des interrelations avec le noyau suprachiasmatique qui est à l'origine des rythmes biologiques endogènes (Moore et Lenn, 1972 ; Moore et Eichler, 1972 ; Moore et Klein, 1974 ; Klein, 1978 ; Rusak et Zucker, 1979). Tous ces faits s'expliquent mal par une simple et unique action orthosympathique provenant des ganglions cervicaux supérieurs et posent le problème d'une innervation plus complexe faisant intervenir des fibres ayant une autre nature et d'autres trajets. 
En fait, l'ablation des ganglions cervicaux supérieurs laisse en place de nombreux filets nerveux dans l'épiphyse chez le rat et le hamster, comme nous avons pu le montrer dès 1975 (Pfister et al.). Ces fibres nerveuses sont myélinisées ou amyéliniques. Elles parviennent à l'épiphyse par le pédicule pinéal et s'arborisent dans la glande entre les pinéalocytes ; leur extrémité distale dégénère après pédiculotomie, ce qui montre bien leur origine centrale.

Pour compléter cette observation, une des possibilités d'étude était de mettre en évidence les péricaryons des neurones d'où proviennent ces fibres. Nous avons utilisé la technique du transport rétroaxonal de la peroxydase de raifort (Kristensson et Olson, 1971, 1973 ; Kuypers, Kievit et Groen-Klevant, 1974 ; Lavail, Winston et Tish, 1973 ; Lavail et Lavail, 1974).

\section{Matériel et méthodes.}

1) Animaux. - Seize rats Sprague-Dawley mâles (souche CRF) de $500 \mathrm{~g}$ ont servi à cette étude. Les animaux sont placés dans des cages individuelles en plastique transparent. Leur régime alimentaire comporte des biscuits spéciaux pour rats et souris (U.A.R.) et de l'eau à volonté. L'animalerie est éclairée artificiellement $14 \mathrm{~h}$ sur 24 , sa température est de $20 \pm 1^{\circ} \mathrm{C}$.

2) Technique de l'injection. - Quatorze rats ont reçu une injection intrapinéale de peroxydase et les deux derniers une simple injection de solution physiologique pour éliminer les erreurs liées à la présence de peroxydase endogène dans le cerveau.

Quinze minutes avant l'intervention, les animaux reçoivent une injection intramusculaire de 0,1 unité Klobusitsky d'hémocoagulase (Reptilase Stago) diluée dans $0,1 \mathrm{ml}$ de solution physiologique pour limiter les hémorragies.

Les animaux sont anesthésiés profondément au Rectanol (tribromoéthanol) à raison de $25 \mathrm{mg}$ pour $100 \mathrm{~g}$ de poids corporel. Les os pariétaux et l'os occipital sont soigneusement dégagés par incision au scalpel et écartement de la peau recouvrant la voûte crânienne. Un volet osseux encadrant le lambda est alors détaché après trépanation avec une fraise et une scie circulaire de dentiste. Le premier de ces instruments permet de pratiquer dans les os crâniens quatre orifices, qui sont ensuite réunis par des traits de scie. Il faut prendre soin de léser le moins possible les méninges sous-jacentes. Après ablation de l'os et arrêt de l'hémorragie due à la trépanation, une ouverture est faite dans les méninges afin de dégager la face postéro-supérieure du cerveau. La partie antérieure du volet méningé ainsi découpé est réclinée vers l'avant et sa partie postérieure vers l'arrière. Cette manipulation permet, en principe, d'arrêter en partie la forte hémorragie due à la section du sinus veineux et de repérer la glande pinéale recouverte par son enveloppe leptoméningée contenant les nerfs conaires. L'innervation complète de l'épiphyse reste ainsi en place. La tête de l'animal est alors fixée sur un appareil à stéréotaxie légèrement modifié pour recevoir une seringue de Hamilton de $5 \mu \mathrm{l}$; l'aiguille est placée verticalement au-dessus de l'épiphyse et descendue délicatement à l'intérieur de la glande sous contrôle d'une loupe binoculaire. Deux à trois microlitres d'une solution de peroxydase de raifort (type VI Sigma) dosée à $0,1 \mu \mathrm{g}$ par $\mu \mathrm{l}$ de solution physiologique sont alors injectés doucement. Afin d'éviter le reflux du liquide, I'aiguille est laissée en place pendant 
1 à 2 min. avant d'être retirée, puis les méninges et le volet osseux sont remis en place. La plaie est refermée grâce à quelques agrafes très soigneusement posées.

La technique d'injection intraépiphysaire est délicate. Les difficultés sont de deux ordres : d'une part, l'hémorragie sourde et continue qu'il faut étancher sans arrêt et qui gêne beaucoup la visibilité ; d'autre part, l'absence de fixité de la glande pinéale. Celle-ci fuit sous l'aiguille au moment de la piqûre et il faut exercer une pression assez forte pour percer la capsule conjonctive qui l'entoure; de ce fait, la glande est très souvent transpercée et il faut alors remonter l'aiguille doucement et au jugé mais, dans de nombreux cas, une partie ou la totalité de la solution de peroxydase est injectée dans le récessus habénulaire du $3^{3}$ ventricule ou même dans le tissu cérébral.

3) Techniques histologiques. - Les animaux sont conservés en vie pendant une durée d'un à trois jours. Ils sont ensuite anesthésiés comme précédemment et sacrifiés par perfusion intra-aortique de $250 \mathrm{ml}$ de formol à $10 \mathrm{p}$. 100 sous débit contrôlé $(400 \mathrm{ml} / \mathrm{h})$. Les ganglions cervicaux supérieurs sont prélevés et le cerveau est soigneusement disséqué. Les pièces sont immergées dans le même fixateur durant 6 à $12 \mathrm{~h}$; elles sont ensuites lavées à l'eau distillée additionnée de 15 p. 100 de glycérol (cryoprotecteur) pendant 24 à $72 \mathrm{~h}$ à $4{ }^{\circ} \mathrm{C}$. Les organes sont débités en totalité au cryostat en coupes sériées de $40 \mu \mathrm{M}$ qui sont déposées sur des lames histologiques préalablement gélatinées. La réaction utilisée pour la détection des peroxydases est celle préconisée par Graham et Karnovsky (1966). Les coupes sont incubées pendant $30 \mathrm{~min}$. dans une solution fraîchement préparée de 3-3' diaminobenzidine (DAB) à l'obscurité $(5 \mathrm{mg}$ de DAB sont dissous dans $10 \mathrm{ml}$ de tampon tris à $\mathrm{pH} 7,6$ additionné de $0,01 \mathrm{ml} \mathrm{de} \mathrm{H}_{2} \mathrm{O}_{2}$ à 10 volumes). Elles sont ensuite rincées rapidement à l'eau distillée et montées au sirop d'Apathy.

\section{Résultats.}

L'examen microscopique a porté sur l'ensemble des coupes sériées de chaque prélèvement. Les animaux témoins ne présentent que de rares structures marquées : en plus des parois vasculaires et des globules rouges, on observe des granules diffus autour du troisième ventricule à la base de l'hypothalamus, ainsi que dans le noyau arqué et le complexe mamillaire. Les animaux ayant reçu de la peroxydase n'ont présenté de marquage significatif que dans quatre cas; les autres ont été éliminés, l'injection ayant dépassé la région pinéale et infiltrant des structures cérébrales ou les cavités ventriculaires. Dans tous les cas positifs, les neurones marqués sont très isolés et peu nombreux : 5 à 20 par cerveau. Le marquage est important dans le cytoplasme du péricaryon et permet de distinguer le départ de l'axone et parfois quelques dendrites.

Chez le premier animal étudié, des cellules colorées par l'enzyme sont mises en évidence dans le colliculus supérieur. Dans le cerveau du $2^{\mathrm{e}}$ rat, $c^{\prime}$ est au niveau de l'habenula, du colliculus supérieur et de l'hypothalamus que les péricaryons marqués ont été repérés. Pour le $3^{\ominus}$ animal, la réaction des peroxydases est visible dans certains neurones de l'amygdale et du colliculus supérieur. Enfin un $4^{\mathrm{e}}$ rat nous a apporté des renseignements plus nombreux, puisque des péricaryons con- 

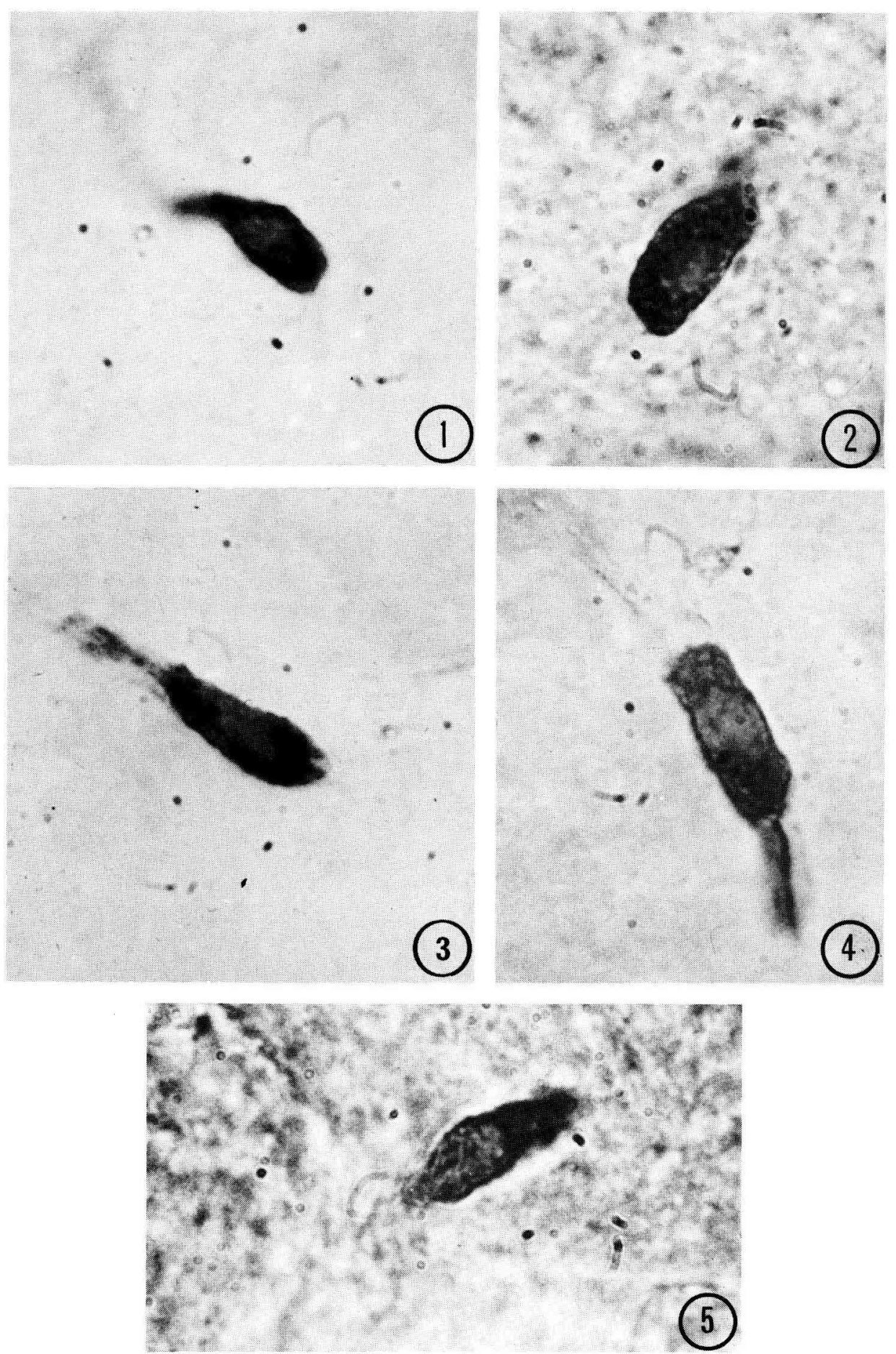
tenant la peroxydase sont présents dans l'amygdale, l'hypothalamus (noyau paraventriculaire gauche, noyau suprachiasmatique, aire préoptique) et la région du centre olfactif.

Les 10 animaux chez lesquels l'injection de peroxydase n'a pas atteint l'épiphyse présentent le plus souvent des lésions traumatiques et hémorragiques au niveau d'un hémisphère cérébral ou du colliculus supérieur. L'étude histologique de leur cerveau révèle le marquage de groupes importants de neurones dans la cicatrice ou à son voisinage et souvent autour des cavités ventriculaires et dans le cortex superficiel. Ces aspects sont très différents de ceux observés lorsque l'injection a réussi, où les neurones marqués sont très rares, très isolés et localisés surtout dans les régions basales du cerveau.

\section{Discussion.}

Les résultats décrits dans ce travail doivent d'abord être justifiés car le nombre des neurones marqués peut paraître faible compte tenu du nombre important de fibres nerveuses efférentes qui existent dans le pédicule pinéal. Cette insuffisance est liée avant tout à la technique : le transport rétrograde de la peroxydase n'est vraiment efficace que dans des fibres nerveuses lésées; or, l'aiguille de la seringue de Hamilton ne transperce que peu d'axones en pénétrant dans l'épiphyse. Ce fait permet aussi d'expliquer la dispersion des résultats d'un animal à l'autre, les éléments touchés étant forcément différents dans chaque expérience. Les localisations relevées se recoupent cependant et sont toujours situées dans les centres neurovégétatifs hypothalamiques ou dans les aires d'association sensorielles. Les deux animaux témoins et les dix chez qui l'injection avait échoué ne présentaient aucun neurone marqué dans ces régions, mais il faut néanmoins remarquer que des neurones à peroxydase endogène ont été signalés chez le rat par Keefer et Christ (1976) au niveau de l'hypothalamus en bordure du noyau suprachiasmatique, dans le noyau supraoptique, le noyau habénulaire et la partie caudale des noyaux périventriculaires. Toutes ces réserves étant faites, il nous a paru utile de présenter ces résultats, encouragés par les travaux électrophysiologiques et surtout par la récente publication de Korf et Wagner (1980) qui, dans des conditions expérimentales voisines des nôtres, ont trouvé des neurones marqués dans les mêmes régions de l'hypothalamus du cobaye.

Ces travaux apportent une contribution à la connaissance des connexions nerveuses directes entre le cerveau et la glande pinéale chez les mammifères. Cette question est toujours en discussion depuis la description de l'innervation de l'épiphyse par Kappers. Cet auteur pensait que les fibres d'origine pédiculaire

FIG. 1 à 5. - Péricaryons marqués par la peroxydase et observés en divers endroits du cerveau.

Fig. 1. - Noyau paraventriculaire droit. Fig. 2. - Noyau paraventriculaire droit. Fig. 3. - Aire préoptique gauche. Fig. 4. - Aire préoptique gauche. Fig. 5. - Amygdale. 
observées dans la portion proximale de l'épiphyse du rat étaient des éléments aberrants provenant des commissures habénulaire et postérieure (Kappers, 1960, 1965). Mais des études morphologiques et électrophysiologiques plus récentes suggèrent l'existence de connexions nerveuses directes entre le cerveau et l'organe pinéal des mammifères. Dès 1973, David et Herbert décrivent une innervation pinéale provenant de la région habénulaire chez le furet. En 1975, les travaux de M $\phi$ ler, Mфllgard et Kimble, ceux de Nielsen et Møller montrent des relations entre l'épiphyse et l'organe sous-commissural chez les fœtus de lapin et de brebis, ainsi que chez des fœtus humains. De même, Lin, Hwang et Tseng (1975) observent la présence de fibres myélinisées et amyéliniques en étudiant I'ultrastructure des glandes pinéales de hamsters gangliectomisés ; ils émettent l'hypothèse d'une origine non sympathique de ces fibres. Des résultats sont rapportés à la même époque par notre équipe après observation en microscopie électronique de l'épiphyse de rats et de hamsters gangliectomisés (Pfister et al., 1975, 1978). En 1979, Rønnekliev et Møller montrent que de petites lésions unilatérales de l'aire habénulaire provoquent la dégénérescence de 15 p. 100 des terminaisons nerveuses pinéales chez le rat.

Les expériences d'électrophysiologie, en particulier celles de McClung et Dafny (1975), de Dafny (1977) mettent en évidence une augmentation de l'inhibition de l'activité électrique spontanée de la pinéale sous divers stimuli. En effet, une inhibition apparaît non seulement après des stimulations photiques, mais aussi acoustiques et également après stimulation directe du tractus optique, de l'amygdale, de l'habenula et du faisceau médian du télencéphale. D'autres équipes confirment ces premiers résultats et les complètent. En 1978, Semm et Vollrath signalent l'existence d'une population de pinéalocytes répondant à des stimuli à court terme. Rønnekliev, Kelly et Wuttke (1980) mettent en évidence la présence de deux populations de fibres pinéales d'origine centrale ; la première, silencieuse et dite habénulo-pinéale, est activée uniquement par une stimulation habénulaire ; la seconde est formée de fibres spontanément actives dont l'origine est variée. Des relations habénulo-pinéales, mises en évidence à la fois par la morphologie et l'électrophysiologie, sont également décrites par Semm, Schneider et Vollrath (1981). Enfin, les travaux électrophysiologiques les plus récents de Dafny (1980 a, b) démontrent que les réponses photiques enregistrées au niveau de la pinéale sont transmises par deux voies séparées ; la première voie, ou voie rapide, a un temps de latence bref et passe par le système nerveux central, le complexe des commissures habénulaire et postérieure et le pédicule épiphysaire ; la seconde voie ou voie lente est celle qui est décrite classiquement et qui passe par les ganglions cervicaux supérieurs.

Les résultats que nous décrivons ici confirment nos travaux précédents sur l'innervation non orthosympathique de l'épiphyse et ceux des auteurs que nous venons de citer. La nature des fibres et des neurones mis en évidence nous est partiellement connue par certaines de nos observations antérieures (Guérillot et al., 1979). En effet, chez les rats gangliectomisés nous avons décrit de grosses fibres acétylcholinestérase-positives dans le pédicule pinéal et dans la partie rostrale de l'épiphyse superficielle, ainsi que des fibres fines et isolées. Un grand nombre de ces fibres persistent dans toute la glande après gangliectomie, ce qui 
tendrait à prouver qu'elles sont de nature cholinergique. D'autres fibres afférentes sont riches en GABA. La valeur fonctionpelle de ces éléments nerveux semble de plus en plus certaine, étant donné la constance et la multiplicité des observations morphologiques et les résultats de l'électrophysiologie, mais leur rôle réel reste encore à découvrir.

Il est possible d'émettre l'idée que de nombreuses informations sensorielles intégrées au niveau du système nerveux central sont réfléchies directement vers l'épiphyse, qui peut ainsi moduler sa réponse en fonction de stimuli variés provenant du milieu extérieur. La glande pinéale ne serait donc pas uniquement soumise à des stimulations orthosympathiques rythmées par les périodes d'éclairement, mais subirait également l'influence des centres neurovégétatifs, des centres sensoriels olfactifs et auditifs et des zones cérébrales commandant le comportement. A toutes ces données, il faut ajouter la réceptivité de la glande aux hormones hypothalamo-hypophysaires et périphériques transitant dans le sang. Ainsi, ce petit organe a tous les éléments suffisants pour intervenir dans la régulation très fine des grandes fonctions végétatives. La complexité de ses interrelations neurohumorales explique les difficultés des recherches consacrées à sa physiologie.

Reçu en juillet 1981.

Accepté en novembre 1981.

\section{Références}

BURKS T. F., DAFNY N., 1977. Morphine and 5-hydroxytryptamine interaction in rat hypothalamus and pineal body. Exp. Neurol., 55, 458-468.

DAFNY N., 1977. Electrophysiological evidence of photic, acoustic and central input to the pineal body and hypothalamus. Exp. Neurol., 55, 449-457.

DAFNY N., 1980 a. Two photic pathways contribute to pineal evoked responses. Life Sci., 26, 737-742.

DAFNY N., 1980 b. Visual input to rat pineal. Experientia (Basel), 36, 1331-1332.

DAVID G., HERBERT J., 1973. Experimental evidence for a synaptic connection between habenula and pineal ganglion in the ferret. Brain Res., 64, 327-333.

GRAHAM R. C., KARNOVSKY M. J., 1966. The early stages of absorption of injected horseradish peroxidase in the proximal tubules of mouse kidney : ultrastructural cytochemistry by a new technique. J. Histochem. Cytochem., 11, 291-302.

GUĖRILLOT C., LEFFRAY P., PFISTER A., DA LAGE C., 1979. Contribution to the study of the pineal stalk nerve fibres in the rat, 97-102. In J. ARIËNS KAPPERS, P. PEVET, The pineal gland of vertebrates including man. Elsevier/North-Holland biomed. Press.

KAPPERS J. A., 1960. The development, topographical relations and innervation of the epiphysis cerebri in the albino rat. $Z$. Zellforsch., 32, 163-215.

KAPPERS J. A., 1965. Survey of the innervation of the epiphysis cerebri and the accessory pineal organs of vertebrates. In J. ARIENS KAPPERS, J. P. SCHADE, Structure and functions of the epiphysis cerebri. Progress in Brain Research. Vol. 10, 87-153, Elsevier/Amsterdam.

KEEFER D. A., CHRIST J. F., 1976. Distribution of endogenous diaminobendizine-staining cells in the normal rat brain. Brain Res., 116, 312-316.

KLEIN D. C., 1978. The pineal gland: a model of neuroendocrine regulation, 303-327. In S. REICHLIN, R. J. BALDESSARIM, J. B. MARTIN, The hypothalamus. Raven Press, New York.

KORF H. W., WAGNER U., 1980. Evidence for a nervous connection between the brain and the pineal organ in the guinea-pig. Cell Tiss. Res., 209, 505-510. 
KRISTENSSON K., OLSON Y., 1971. Retrograde axonal transport of protein. Brain Res., 29, 363-365.

KRISTENSSON K., OLSON Y., 1973. Uptake and retrograde axonal transport of protein tracers in hypoglossal neurons. Fate of a tracer and reaction of the nerve cell bodies. Acta neuropathol., 23, 43-47.

KRISTENSSON K., OLSON Y., 1973. Diffusion pathways and retrograde axonal transport of protein tracers in peripheral nerves. Progr. Neurobiol., 1, 85-109.

KUYPERS H. G. J. M., KIEVIT J., GOREN-KLEVANT A. D., 1974. Retrograde axonal transport of horseradish peroxidase in rat's forebrain. Brain Res., 67, 211-218.

LAVAIL J. H., LAVAIL M. M., 1974. Retrograde axonal transport in the central nervous system. Science, 176, 1416-1417.

LAVAIL J. H., WINSTON K. R., TISH A., 1973. A method based on retrograde axonal transport of protein for identification of cell bodies of origin of axons terminating within the central nervous system. Brain Res., 58, 470-477.

LIN H. S., HWANG B. H., TSENG C. Y., 1975. Fine structural changes in the hamster pineal gland after blinding and superior cervical ganglionectomy. Cell Tiss. Res., 158, 285-299.

MCCLUNG R., DAFNY N., 1975. Neurophysiological properties of the pineal gland. II. Single unit recording. Life Sci., 16, 621-628.

McKINNEY T. D., VAUGHAN M. K., REITER R. J., 1975. Pineal influence on intermale agression in adult house mice. Physiol. Behav., 15, 213-216.

MILINE R., DEVEČERSKI V., KRSTIC R., 1963. Influence d'excitations olfactives sur le système habénulo-épiphysaire. Ann. Endocrinol., 24, 377-379.

M $\emptyset$ LLER M., M $\emptyset$ LLGARD K., KIMBLE E., 1975. Presence of a pineal nerve in sheep and rabbit fetuses. Cell Tiss, Res., 158, 451-459.

MOORE R. Y., LENN N. J., 1972. A retino-hypothalamic projection in the rat. J. comp. Neurol., 146, $1-14$.

MOORE R. Y., EICHLER V. B., 1972. Loss of a circadian adrenal corticosterone rhythm following suprachiasmatic lesions in the rat. Brain Res., 42, 201-206.

MOORE R. Y., KLEIN D. L., 1974. Visual pathways and the central neural control of a circadian rhythm in pineal serotonin $\mathrm{N}$-acetyltransferase activity. Brain Res., 71, 17-33.

NIELSEN J. I., MØLLER M., 1975. Nervous connections between the brain and the pineal gland in the cat (Felis catus) and the monkey (Cercopithecus aethiops). Cell Tiss. Res., 161, 293301.

PFISTER A., GUÉRILLOT C., MÜLLER J., VENDRELY E., DA LAGE C., 1975. Existence d'une innervation d'origine centrale dans l'épiphyse du hamster et du rat. J. Physiol. (Paris), 70, 10B (abstract).

PFISTER A., MÜlLeR J., LefFRAY P., GUÉRILLOT C., VENDRELY E., DA LAGE C., 1978. Investigation on a possible extraorthosympathetic innervation of the pineal in rat and hamster. $J$. neur. Transm., suppl. 13, 390-391.

REITER R. J., ELLISON N. M., 1970. Delayed puberty in blinded anosmic female rats : role of the pineal gland. Biol. Reprod., 2, 216-222.

RØNNEKLIEV O. K., M $\emptyset$ LLER M., 1979. Brain-pineal nervous connections in the rat : an ultrastructural study following habenular lesion. Exp. Brain Res., 37, 551-562.

RфNNEKLIEV O. K., KELLY M. J., WUTTKE W., 1980. Single unit recordings in the rat pineal gland : evidence for habenulo-pineal neural connections. Exp. Brain Res., 39, 187-192.

RUSAK B., ZUCKER I., 1979. Neural regulation of circadian rhythms. Physiol. Rev., 59, 449-526.

SEMM P., VOLLRATH L., 1978. Electrophysiological properties of single cells of the guinea pig epiphysis cerebri. Pflügers Arch., suppl. 373, R 55.

SEMM P., SCHNEIDER T., VOLLRATH L., 1981. Morphological and electrophysiological evidence for habenular influence on the guinea-pig gland. $J$. neur. Trans., 50, 247-266. 